seventh $\sin$ ) is different altogether. Persistence refers to the retention of highly emotional memories that one would rather forget. This independence of mechanism means that a treatment, such as a drug, developed to help 'memory' is unlikely to work on all of memory's imperfections.

Schacter's framework of research findings and interpretations, organized around his seven categories, succeeds in describing a discipline of work, theoretical and applied, that is both fascinating and of practical importance. The tip-of-the tongue phenomenon (the feeling that a momentarily unavailable word or name is on the tip of the tongue) is experienced so similarly across cultures that 45 of 51 languages surveyed use expressions containing the word 'tongue' to describe this psychological state (including Estonian, Cheyenne and Afrikaans). What is commonly termed absent-mindedness (the second $\sin$ ) is due to a disconnection between attention and memory, for example, a failure to attend at the moment when information is encoded. Absent-mindedness also occurs when retrieval cues are not available or are not noticed when an intended action is meant to be carried out.

Another imperfection involves the tendency to misremember the past so as to make it more consistent with current knowledge and beliefs (bias, the sixth sin). College students who were dating were asked about their partners on different occasions two months apart. Those whose evaluations become more negative during the two months recalled their original impressions as more negative than they actually were. Conversely, those whose evaluations became more positive recalled their original impressions as more positive than they actually were.

During the past decade, the fragility of memory became a prominent issue in the courtroom and in the news. Here, the guilty features of memory are misattribution (the fourth sin) and suggestibility (the fifth sin). Misattribution refers to errors in which a thought or a dream or a general feeling of familiarity is reported as a real memory. Suggestibility refers to distortions of memory through leading questions or other forms of influence. It is easy to demonstrate in the laboratory how readily individuals will succumb to memory errors of these kinds. After seeing a word list (bed, rest, awake, tired, dream, wake, snooze, blanket, doze, slumber, snore, nap, peace, yawn, drowsy), individuals frequently report with confidence that a highly associated word (sleep) was on the list. Persons with a capacity for vivid imaging, who score high on suggestibility scales, show a greater tendency for this effect.

There are many examples where misattribution and suggestibility appear to be at work. These include the corruptibility of young children's memories in the face of leading questions, false confessions, early memories of abuse that were 'recovered' during psychotherapy (and later disavowed), and eyewitness misidentification. It is heartening to see that newer studies reviewed by Schacter are having practical consequences. For example, line-up identification procedures have been improved in some places by the simple expedient of presenting suspects one at a time and asking for a yes-no judgement. In the traditional method, the observer makes a choice after seeing all the suspects, but is vulnerable to error when the culprit is not in the line-up, because of a tendency to select the person who looks most like the culprit.

This book brings together an enormous amount of material in a form that is entertaining, informative and credible. It can both be enjoyed by the general reader and used as a resource by the specialist, as the text is accompanied by 19 pages of notes and citations supporting its empirical statements. Lawyers, judges, health-care professionals and others whose work sometimes relies on intensely practical questions about memory should also find it a welcome source of information.

Larry R. Squire is at the Veterans Affairs Healthcare System and in the Departments of Psychiatry, Neurosciences and Psychology, University of California, San Diego, California 92161, USA.

\section{. A physical view of the cytoskeleton}

Mechanics of Motor Proteins and the Cytoskeleton

by Jonathon Howard

Sinauer/Palgrave: 2001. 384 pp.

$\$ 59.95 / £ 44.99$

\section{Edwin Taylor}

The cytoskeleton consists of three kinds of fibrous protein filament - actin filaments, intermediate filaments and microtubules plus the proteins that interact with them. The molecular motors myosin, kinesin and dynein run along the filament tracks. At least 100 other proteins interact with the cytoskeleton and new ones continue to be discovered.

The cytoskeleton is an area of intense research and we are in danger of drowning in a sea of facts. What should we try to teach our students about it? Popular textbooks devote one or two chapters to the cytoskeleton and motility and they can be supplemented by a book such as Dennis Bray's Cell Movements (Garland, 2001). But a textbook is needed which starts from first principles and leads to an understanding of the dynamics of the system.
And here is that book. In the first part, Joe Howard presents the physical principles that govern the behaviour of the cytoskeleton. It is a mechanical system composed of thin rods that are under stress. Movements occur in a medium in which inertia is negligible compared to viscous damping. The basic problem is the motion of a small elastic body in a viscous medium subject to an applied force and thermal motion. This problem is presented in a clear, conversational style and the level is that of a first-year college physics course in which some calculus is needed.

It may seem surprising that important properties of fibrous proteins and motors can be deduced from their resistance to stretching and bending, but proteins are large enough to be treated as bits of continuous matter. Howard points out that the elastic properties of these proteins have very little to do with the amino-acid sequence or even with the three-dimensional structure, because the proteins are held together by the same kinds of weak bonds.

Ask a student or colleague how long it takes a protein of relative molecular mass 100,000 to diffuse 1 millimetre, or what is the rotational time of a myosin crossbridge (or any other protein of similar shape). The chances are that the answers will be wrong by a few orders of magnitude. By including many simple calculations, some left for the reader to do, Howard succeeds in building up a feeling for the dynamics of the cytoskeleton.

The results derived in the first part of the book are then used to treat the mechanical properties of the cytoskeleton. The molecular motors myosin and kinesin are discussed in the third and final part. Howard is a major contributor to studies of kinesin, and provides a clear description of the techniques of mechanical measurements on single molecules.

This book would be an excellent text for a semester course on the cytoskeleton and motility. Most of the topics are accessible to students who have had a course in physics. Some topics in diffusion and thermal motion use more advanced mathematics, but the solutions to equations have been wisely placed in an appendix where they don't get in the way of the reader who is interested in results, not mathematical derivations. It will also be a valuable reference book for researchers in the motility field.

Students on general cell-biology courses often have an aversion to equations, but a selection of some of the results discussed in this book would be a valuable supplement to such a course.

Edwin Taylor is in the Department of Molecular

Genetics and Cell Biology, University of

Chicago, 920 East 58th Street, Chicago,

Illinois 60637, USA. 